\title{
NgAgo-based fabp11a gene knockdown causes eye developmental defects in zebrafish
}

Cell Research (2016) 26:1349-1352. doi:10.1038/cr.2016.134; published online 11 November 2016

\section{Dear Editor,}

A recent report of genome editing using Natronobacterium gregoryi Argonaute ( $\mathrm{NgAgo}$ ) with guide DNA (gDNA) in human cells [1] prompted us to explore the utility of this protein for in vivo genetic manipulation in zebrafish (Danio rerio). Zebrafish is a model organism that offers several distinct advantages for studying genetics, developmental biology, vascular biology and disease modeling. In the last several years, loss-of-function genomic editing techniques, including zinc-finger nucleases (ZFNs) [2, 3], artificial transcription activator-like effector nucleases (TALENs) $[4,5]$ and clustered regularly interspaced short palindromic repeats (CRISPR)/ CRISPR-associated (Cas) 9 system (CRISPR/Cas9) [6, 7], have been adopted for zebrafish. Recently the gDNA/ NgAgo system has elicited much interest because of some unique advantages: low tolerance to guide-target mismatch, minimum off-target effects, and easy to design [1]. Here we investigated whether the gDNA/NgAgo system could be used to manipulate zebrafish genes in vivo using fabplla as a test case.

Fatty acid binding protein 11a (Fabp11a) is a member of the family of intracellular FABPs, which play important roles in regulating glucose and lipid homeostasis as well as inflammation $[8,9]$. So far, the role of Fabp11a in zebrafish embryonic development remains unclear. To investigate whether 5 '-phosphorylated single-strand (ss) DNA can direct NgAgo to disrupt endogenous genes in vivo in zebrafish, we constructed a codon-optimized $\mathrm{NgAgo}$ for zebrafish with nuclear localization signal (NLS) peptides at both $\mathrm{N}$-terminus and $\mathrm{C}$-terminus (NgAgo-2nls, Figure 1A and Supplementary information, Data S1). Two 24 bp 5'-phosphorylated ssDNA oligos, forward guide-1 (FW-guide 1) and FW-guide 2 (Supplementary information, Table S1A, which contains other gDNAs used in this study), targeting the second exon and third exon of fabp 11a gene (Figure 1B) were synthesized; and each was coinjected with $\mathrm{NgAgo-2nls} \mathrm{mRNA}$ into 1-cell stage embryos (Figure 1A). Interestingly, we observed that approximately $30 \%$ of the injected embry- os displayed severe eye phenotypes, either having one very small eye and one relative normal size eye (type 1 ), or having one large fused eye on the top of the head like Cyclops (type 2), at 30 hours post fertilization (hpf) (Figure 1C and 1D, Supplementary information, Figure $\mathrm{S} 1 \mathrm{~A}(\mathrm{~A}-\mathrm{C})$ ).

To investigate whether the eye phenotype was caused by genetic mutations in fabp $11 a$ gene, we extracted genomic DNA from embryos with abnormal eye phenotype and performed polymerase chain reaction (PCR) amplification of the targeted region. Unexpectedly, Sanger sequencing did not uncover any DNA sequence alteration in the targeted region from 57 embryos with eye phenotype we analyzed (data not shown). Instead of DNA mutations, real time reverse transcriptase (RT)PCR analysis revealed that the fabp 11a gene expression was down-regulated (Figure 1E and Supplementary information, Figure S1A(D)). In contrast, we did not find any specific phenotype in only $\mathrm{NgAgo-2nls} \mathrm{mRNA-} \mathrm{or}$ gDNA-injected embryos (Figure 1D), suggesting that the phenotype was caused by gDNA/NgAgo complex. Coinjection of $\mathrm{NgAgo-2nls} \mathrm{mRNA} \mathrm{with} \mathrm{two} \mathrm{individual}$ mismatched oligos (Supplementary information, Table S1) did not cause the eye developmental defect (Figure 1D and data not shown), suggesting specificity of the gDNA/NgAgo system. We further designed two reversed gDNAs: RV-guide 1 and 2 complementary to FW-guide 1 and FW-guide 2, respectively. Coinjection of RV-guide 1 or 2 with $\mathrm{NgAgo-2nls} \mathrm{mRNA} \mathrm{into} \mathrm{1-cell} \mathrm{stage} \mathrm{embryos}$ resulted in abnormal eye development in approximately $15 \%$ of embryos (Figure 1D and data not shown). Real time RT-PCR results revealed the reduction of fabp $11 a$ expression (Figure 1E). The abnormal eye phenotype caused by gDNA/NgAgo system could be rescued by coinjection of fabp 11 a mRNA (Figure 1D).

To confirm that gDNA/NgAgo system could knock down genes as a general principle in zebrafish, we tested another gene ta (no tail/ntl or brachyury). ta encodes an essential T-box transcription factor required for proper mesoderm formation; zebrafish embryos homozygous for a null ta mutation lack the notochord and tail [10, 


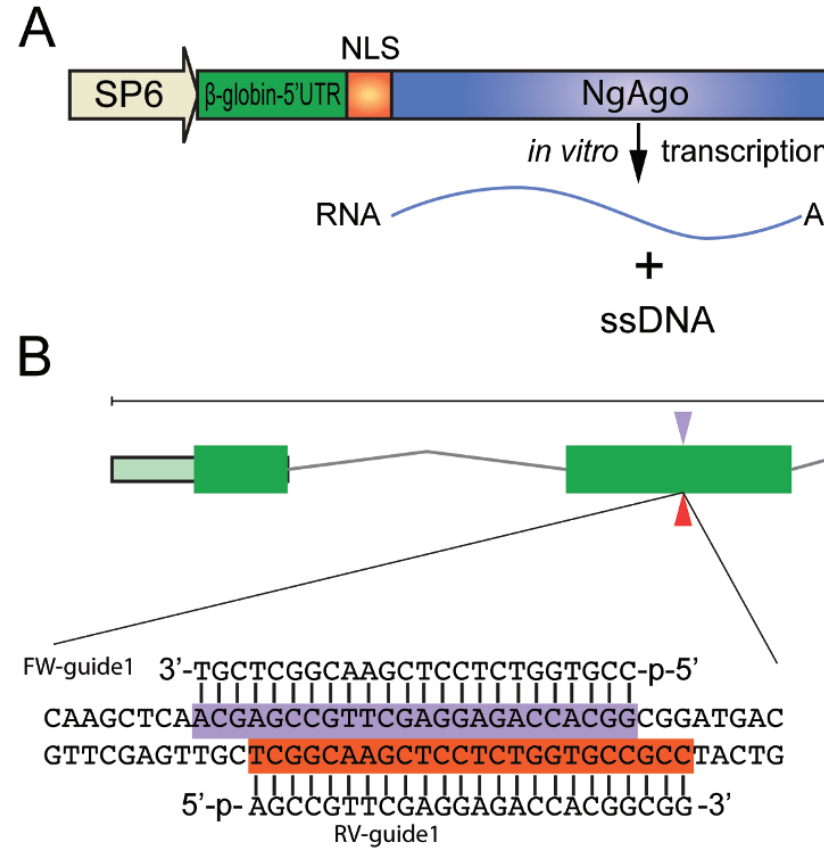

NLS
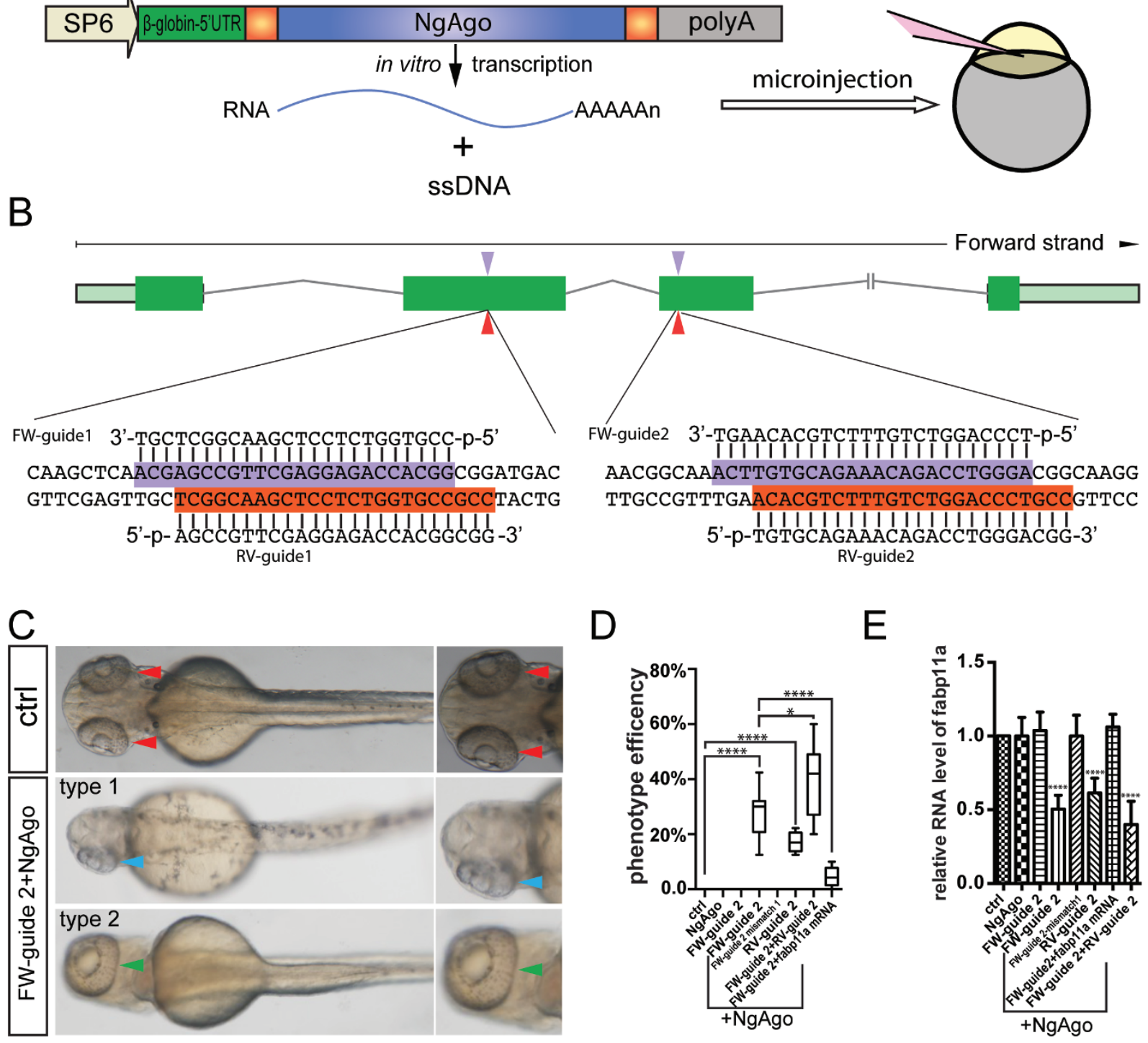

Figure $1 \mathrm{NgAgo-based} \mathrm{fabp11a} \mathrm{gene} \mathrm{knockdown} \mathrm{causes} \mathrm{eye} \mathrm{developmental} \mathrm{defects} \mathrm{in} \mathrm{zebrafish.} \mathrm{(A)} \mathrm{Schematic} \mathrm{diagram}$ showing the structure of $\mathrm{NgAgo}-2 \mathrm{nls}$ for in vitro transcription and microinjection. (B) Schematic diagram showing guide DNA targeting sites (indicated by arrowhead) in the exons of fabp11a gene. The targeting sequences are highlighted in purple and red. (C) Microscopy analysis of eyes in control and FW-guide DNA 2 and NgAgo-2n/s mRNA coinjected embryos, dorsal view, with the head regions enlarged and shown on the right. Red arrowheads indicate normal paired eyes. Blue arrowhead indicates one single eye in FW-guide 2 and NgAgo-2n/s mRNA coinjected embryo. Green arrowhead indicates a single fused large eye. (D) Statistical analysis of phenotype efficiency in control (0\%); NgAgo-2n/s mRNA only (0\%); FW-guide 2 only (0\%); FW-guide 2 and NgAgo-2n/s mRNA (29.6\%); FW-guide 2 mismatch 1 and NgAgo-2nls mRNA (0\%); RV-guide 2 and NgAgo-2nls mRNA (15.4\%); FW-guide 2, RV-guide 2 and NgAgo-2nls mRNA (39.7\%); FW-guide 2, NgAgo-2nls mRNA and fabp11a mRNA injected embryos (4.6\%). One-Way ANOVA; ${ }^{* * *} P<0.0001 ;{ }^{*} P<0.05$. (E) Relative mRNA levels of zebrafish fabp11a in 30 hpf control; NgAgo-2nls mRNA only; FW-guide 2 only; FW-guide 2 and NgAgo-2nls mRNA; FW-guide 2 mismatch 1 and NgAgo-2nls mRNA; RV-guide 2 and NgAgo-2nls mRNA; FW-guide 2, NgAgo-2nls mRNA and fabp11a mRNA; FW-guide 2, RV-guide 2 and NgAgo-2n/s mRNA injected embryos. One-Way ANOVA; ${ }^{* * * *} P<0.0001$.

11]. Two 24 bp gDNAs were individually injected with NgAgo-2nls mRNA into 1-cell stage embryos; and, at 30 hpf, around $30 \%$ injected embryos showed typical no tail phenotype, whereas coinjection of mismatched gDNAs with $\mathrm{NgAgo-2nls}$ mRNA resulted in normal embryos (Supplementary information, Figure S1B and data not 
shown). 40 of the no tail-like embryos were analyzed for insertion/deletion (indel). We did not detect any mutation in the target region by Sanger sequencing (data not shown). Real time RT-PCR revealed that $t a$ expression was down-regulated (Supplementary information, Figure S1B(D)). Furthermore we tested three more genes, $k d r l$, lamal and ftt1. The gDNA/NgAgo system significantly reduced the expression of these genes and caused specific phenotypes similar to previously reported [12-14] (Supplementary information, Figure S1C-S1E).

To test whether the length of guide DNA can influence knockdown efficiency, we used gDNAs of various lengths (20-27 nt) to target fabp 11a and ta. We found that the shorter gDNAs displayed higher knockdown efficiency for both genes (Supplementary information, Figure S1F).

We also investigated whether NgAgo could generate indels under certain conditions. We injected NgAgo mRNA mixed with two complementary gDNAs targeting fabp11a and found that the injection caused abnormal eye phenotype but failed to introduce indels (Figure 1D and $1 \mathrm{E})$. Recent report showed that gDNA/NgAgo could generate $11.2 \%-41.3 \%$ indels in human cells at $37^{\circ} \mathrm{C}$ [1], whereas in our experiments described above the zebrafish were maintained at $28.5{ }^{\circ} \mathrm{C}$. Temperature might be a reason that influences nuclease activity of $\mathrm{NgAgo}$. It has been reported that incubation at a temperature above 32.5 ${ }^{\circ} \mathrm{C}$ may cause malformations of the zebrafish embryo [15]. We found more than half of the embryos developed normally when they were incubated at $37{ }^{\circ} \mathrm{C}$ continuously until phenotypic examination. Under this condition, gDNA/NgAgo injection still failed to introduce indels in fabplla in 38 embryos exhibiting eye phenotype and in ta in 50 embryos exhibiting no tail-like phenotype (data not shown). Additionally, we mutagenized two aspartic acid residues in NgAgo obtaining NgAgo-D663A, NgAgo-D738A and NgAgo-D663A-D738A, which are predicted to lack the catalytic activity of NgAgo. Coinjection of each mutant $\mathrm{NgAgo}$ mRNA with fabp lla FW-gDNA2 efficiently caused the eye developmental defect, with phenotype efficiency of $28.7 \%, 29.5 \%$ and $23.9 \%$, respectively (Supplementary information, Figure S1G(A)). Coinjection of the mutants with $t a$ FW-gDNA1 caused no tail-like phenotype with efficiency of $32.5 \%$, $33.8 \%$ and $29.8 \%$, respectively (Supplementary information, Figure $\mathrm{S} 1 \mathrm{G}(\mathrm{B}))$. We could not detect any indel either in fabplla in $25 \mathrm{NgAgo-D663A-D738A} \mathrm{mRNA-} \mathrm{and}$ fabp 11a FW-gDNA 2-injected embryos exhibiting eye phenotype, or in $t a$ in $25 \mathrm{NgAgo-D663A-D738A} \mathrm{mRNA-}$ and ta FW-gDNA 1-injected embryos exhibiting no taillike phenotype.

To confirm that the eye developmental defect was caused by fabp 11a knockdown, we carried out further investigation. First, we examined the expression pattern of fabp 11a at early developmental stages of zebrafish embryos. Whole mount in situ hybridization (WISH) analysis demonstrated that fabp 11a is specifically and highly expressed in the eye field (optic primordium) (Supplementary information, Figure $\mathrm{S} 1 \mathrm{H}$ ), suggesting fabp 11a plays important roles in eye development from an early stage. We next knocked down fabp 11a using a translation-blocking morpholino (Supplementary information, Table S1A), whose efficiency was validated (data not shown); and fabplla morphants showed similar eye defects (Supplementary information, Figure S1I). Interestingly, the knockdown efficiency of gDNA/NgAgo system is higher than that of the morpholino-mediated approach. In addition, we knocked out fabp 11 a using CRISPR/Cas9 system. We used two gRNAs to target fabplla locus, and coinjected cas 9 mRNA with individual gRNA into onecell embryos. Both gRNA1 and gRNA2 efficiently induced indels in fabp $11 a$ locus with efficiency of $60 \%$ and $42.9 \%$, respectively (Supplementary information, Figure S1J(A-D)). gRNA1 resulted in severe eye developmental defect in approximately 30\% embryos (Supplementary information, Figure $\mathrm{S} 1 \mathrm{~J}(\mathrm{E}$ and $\mathrm{F})$ ); about $10 \%$ of total embryos showed extreme eye phenotype with both eyes missing at $48 \mathrm{hpf}$. In addition, the established fabp 11a mutant embryos showed very similar eye developmental defect (Supplementary information, Figure S1J(G)) in the F1 generation, suggesting the fabp 1la mutations and the eye abnormal phenotype are heritable.

In summary, our study shows that gene knockdown is the main mechanism by which gDNA/NgAgo affects gene function in zebrafish. This is supported by results of the experiments using presumably catalytically dead $\mathrm{NgAgo}$. In addition, we have failed to detect any mutation in all the embryos we examined. Since the catalytically inactive (dead) Cas9 (dCas9):sgRNA complex could efficiently inhibit gene expression through binding to the coding sequence [16], we hypothesize that gDNA/ $\mathrm{NgAgo}$ may bind to a target gene to block its transcription. Overall, we suggest that the gDNA/NgAgo system provides an alternative strategy for gene knockdown in zebrafish.

\section{Acknowledgments}

This study was supported by grants from the National Natural Science Foundation of China $(81570447 ; 81370302 ; 31400918$; 31201083 ) and Natural Science Foundation of Jiangsu Province (BK20150404; BK2012228; 12KJB180010).

Jialing $\mathrm{Qi}^{1,{ }^{*}}$, Zhangji Dong ${ }^{1,}{ }^{*}$, Yunwei $\mathrm{Shi}^{1}$, Xin Wang ${ }^{1}$, 


\author{
Yinyin Qin ${ }^{1}$, Yongming Wang ${ }^{2}$, Dong Liu ${ }^{1}$ \\ ${ }^{I}$ Co-innovation Center of Neuroregeneration, Jiangsu Key Laboratory of \\ Neuroregeneration, Nantong University, Nantong, Jiangsu 226001, China; \\ ${ }^{2}$ The State Key Laboratory of Genetic Engineering and MOE Key Labora- \\ tory of Contemporary Anthropology, School of Life Sciences, Fudan Uni- \\ versity, Shanghai 200433, China \\ *These two authors contributed equally to this work. \\ Correspondence: Dong Liu \\ E-mail: liudongtom@gmail.com; tom@ntu.edu.cn
}

\section{References}

Gao F, Shen XZ, Jiang F, et al. Nat Biotechnol 2016; 34:768-773.

2 Gupta A, Christensen RG, Rayla AL, et al. Nat Methods 2012; 9:588590.

3 Zhu C, Smith T, McNulty J, et al. Development 2011; 138:4555-4564.

4 Huang P, Xiao A, Zhou M, et al. Nat Biotechnol 2011; 29: 699-700.

5 Bedell VM, Wang Y, Campbell JM, et al. Nature 2012; 491:114-118.

6 Hwang WY, Fu Y, Reyon D, et al. Nat Biotechnol 2013; 31:227-229.

7 Chang N, Sun C, Gao L, et al. Cell Res 2013; 23:465-472.

8 Makowski L, Boord JB, Maeda K, et al. Nat Med 2001; 7:699-705.

9 Furuhashi M, Tuncman G, Görgün CZ, et al. Nature 2007; 447:959965.
10 Halpern ME, Ho RK, Walker C, et al. Cell 1993; 75:99-111.

11 Doyon Y, McCammon JM, Miller JC, et al. Nat Biotechnol 2008; 26:702-708.

12 Covassin LD, Siekmann AF, Kacergis MC, et al. Dev Biol 2009; 329:212-226.

13 Pollard SM, Parsons MJ, Kamei M, et al. Dev Biol 2006; 289:64-76.

14 Krueger J, Liu D, Scholz K, et al. Development 2011; 138:2111-2120.

15 Pype C, Verbueken E, Saad MA, et al. Reprod Toxicol 2015; 56:5663.

16 Qi LS, Larson MH, Gilbert LA, et al. Cell 2013; 152:1173-1183.

(Supplementary information is linked to the online version of the paper on the Cell Research website.)

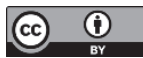

This work is licensed under a Creative Commons Attribution 4.0 Unported License. The images or other third party material in this article are included in the article's Creative Commons license, unless indicated otherwise in the credit line; if the material is not included under the Creative Commons license, users will need to obtain permission from the license holder to reproduce the material. To view a copy of this license, visit http:// creativecommons.org/licenses/by/4.0/

(C) The Author(s) 2016 